\title{
Isoproterenol and Lidocaine for Recurrent Torsades de Pointes in a 32-Year-Old Pregnant Woman
}

\author{
Breyanna Reachi, PharmD ${ }^{1 *}$, Jenna Negrelli, PharmD ${ }^{2}$, Abby Hickman, PharmD, MBA, BCCCP ${ }^{1}$, Sarah \\ Beesley, $M D^{3}$ and Jeffrey Osborn, $M D^{4}$
}

${ }^{1}$ Department of Pharmacy, Intermountain Medical Center, USA

${ }^{2}$ Department of Pharmacy, Loyola University Medical Center, USA

${ }^{3}$ Department of Critical Care, Intermountain Medical Center, USA

${ }^{4}$ Department of Electrophysiology, Intermountain Medical Center, USA

*Corresponding author: Breyanna Reachi, PharmD, Department of Pharmacy, Intermountain Medical Center, 5121 S Cottonwood St, Murray, UT 84107, USA, Tel: 505-400-4277

\begin{abstract}
Guidelines recommend magnesium and defibrillation for Torsades de Pointes (TdP) treatment in pregnancy, both of which were unsuccessful at terminating our patient's recurrent ventricular arrhythmias. Both lidocaine and isoproterenol carry potential risks to mother and fetus, but the risk of congenital abnormalities is significantly lower compared to other antiarrhythmics such as amiodarone.

A 15-week pregnant 32-year-old female was admitted to an outside hospital for alcohol withdrawal and developed multiple episodes of ventricular tachycardia (VT) and TdP resulting in her prompt transfer to our hospital for escalation of care. On arrival she was awake, alert, and hemodynamically stable but quickly began experiencing recurrent episodes of TdP that developed into pulseless VT despite defibrillation, magnesium and calcium administration, and chest compressions. We initiated lidocaine and isoproterenol infusions, after which the patient stabilized. Isoproterenol and lidocaine were successfully discontinued after 2 days of treatment and the patient was discharged after 4 days of hospitalization with fetal heart sounds intact.

While there are guideline recommendations for the management of TdP and ventricular arrhythmias, primary literature is lacking. This unique case demonstrates efficacy of isoproterenol pacing combined with the antiarrhythmic lidocaine for terminating a refractory and life-threatening ventricular arrhythmia.
\end{abstract}

\section{Keywords}

Torsades de Pointes, Isoproterenol, Lidocaine, Long QT syndrome, Arrhythmias, Cardiac, Pregnancy

\begin{abstract}
Abbreviations
VT: Ventricular Tachycardia; VF: Ventricular Fibrillation; ACC: American College of Cardiology; AHA: American Heart Association; ESC: European Society of Cardiology; TdP: Torsades de Pointes; CPR: Cardiopulmonary Resuscitation; ICU: Intensive Care Unit; ECG: Electrocardiogram; IV: Intravenous; ms: milliseconds; bpm: beats per minute
\end{abstract}

\section{Introduction}

Polymorphic ventricular tachycardia (VT) and Torsades de pointes (TdP) have a significant impact on health outcomes and can even lead to sudden cardiac death. The 2006 American College of Cardiology/ American Heart Association/European Society of Cardiology (ACC/AHA/ESC) and the 2017 AHA/ACC/Heart Rhythm Society (HRS) guidelines state that pacing, or isoproterenol are reasonable temporary treatments for patients who present with recurrent pause-dependent TdP not suppressed by IV magnesium. Intravenous (IV) lidocaine may be considered for patients who present with VT, ventricular fibrillation (VF), or TdP, especially if unresponsive to defibrillation. The standard of care recommendation for pregnant women is electrical cardioversion or defibrillation [1]. Isoproterenol may shorten the QT interval and refractory period, preventing pause-dependent TdP. Lidocaine has been used effectively in dog and rabbit models to prevent induction of

Citation: Reachi B, Negrelli J, Hickman A, Beesley S, Osborn J (2019) Isoproterenol and Lidocaine for Recurrent Torsades de Pointes in a 32-Year-Old Pregnant Woman. Int J Crit Care Emerg Med 5:092. doi. org/10.23937/2474-3674/1510092

Accepted: September 17, 2019: Published: September 19, 2019

Copyright: (C) 2019 Reachi B, et al. This is an open-access article distributed under the terms of the Creative Commons Attribution License, which permits unrestricted use, distribution, and reproduction in any medium, provided the original author and source are credited. 
polymorphic VT and TdP suppression and prevention in human case reports [2].

There is a lack of primary literature to guide the management of TdP and ventricular arrhythmias. An extensive literature search revealed only a few case reports of lidocaine and isoproterenol being used together for patients experiencing $\mathrm{TdP}$, and a single case report on the use of isoproterenol for TdP in a pregnant female [3-5]. Takaya, et al. reported a case of donepezil-induced TdP in an 81-year-old female, refractory to magnesium and lidocaine, that was successfully treated with isoproterenol [3]. Katz, et al. reported 2 cases of loperamide-induced TdP. The first, a 28-year-old man, was initially responsive to IV magnesium, sodium bicarbonate, lidocaine and isoproterenol $(2-10 \mathrm{mcg} / \mathrm{min})$ but developed persistent TdP when isoproterenol was discontinued for tachyphylaxis and ultimately required a pacemaker. The second, a 39-year-old woman with TdP refractory to IV magnesium and sodium bicarbonate, was successfully treated for 4 days with IV isoproterenol titrated to HR above 100 beats per minute (bpm) [4]. Mittadodla, et al. reported a case of idiopathic VF in a 32-year-old 6-week pregnant woman, refractory to IV lidocaine, metoprolol and amiodarone, that was successfully treated with isoproterenol titrated to a heart rate above $120 \mathrm{bpm}$ [5]. Our unique case of isoproterenol pacing combined with the antiarrhythmic lidocaine for refractory TdP and recurrent VT arrests in a 32-year-old pregnant patient provides additional information for the utilization of these drugs in a specialized population.

\section{Case Description}

A 15-week pregnant 32-year-old female was admitted as a transfer from an outside hospital for escalation of treatment after initially presenting for alcohol withdrawal. She presented after a syncopal event accompanied by urinary incontinence. She reported a three-day drinking binge prior to this event, during which she consumed $750 \mathrm{~mL}$ of vodka per day. She reported that this episode was similar to previous alcohol withdrawal seizures she had experienced in the past.

At the outside hospital she was diagnosed with alcohol induced pancreatitis with a lipase above 4000 units/L and was treated with IV fluids, morphine, and lorazepam. The next morning, she had a single episode of VT accompanied by syncope but did not lose a pulse. A few hours later she had an episode of pulseless VT for which she received 5 minutes of cardiopulmonary resuscitation (CPR), $1 \mathrm{mg}$ of epinephrine, and a single defibrillation before she regained spontaneous circulation. Five hours later she had an additional episode of VT/ TdP which resolved with administration of magnesium. She was started on amiodarone and isoproterenol infusions and transferred to our hospital. Doses were not reported, and isoproterenol was discontinued in route due to tachycardia.

On presentation to our hospital she was awake, alert, and hemodynamically stable. Shortly after admission to the intensive care unit the patient began experiencing additional episodes of TdP that developed into pulseless VT. Her original episode of TdP developed while obtaining her admission electrocardiogram (ECG), which demonstrated multifocal VT converting to TdP and then sinus tachycardia with a heart rate of 180 bpm and a QTc of 641 milliseconds (ms). A 4 gm dose of magnesium IV was administered, and her rhythm progressed to obvious TdP with a reported heart rate on the ECG strip of $235 \mathrm{bpm}$ and a QTc of $352 \mathrm{~ms}$. (Figure 1) This episode resolved with administration of magnesium, but she developed subsequent episodes of non-sustained TdP. Each time her heart rate dropped below 100 bpm she developed ectopy and bigeminy that precipitated VT episodes leading to VF. Once pulseless she received 1 minute of CPR, a $200 \mathrm{~J}$ defibrillation, and another minute of CPR, after which the patient regained a pulse and awoke fully. She also received $1 \mathrm{gm}$ calcium chloride, 4 grams magnesium, $0.1 \mathrm{mg}$ epinephrine, and $40 \mathrm{mEq}$ potassium chloride during this episode. The electrophysiology team recognized the ectopic pauses precipitating each VT event and lidocaine and isopro-

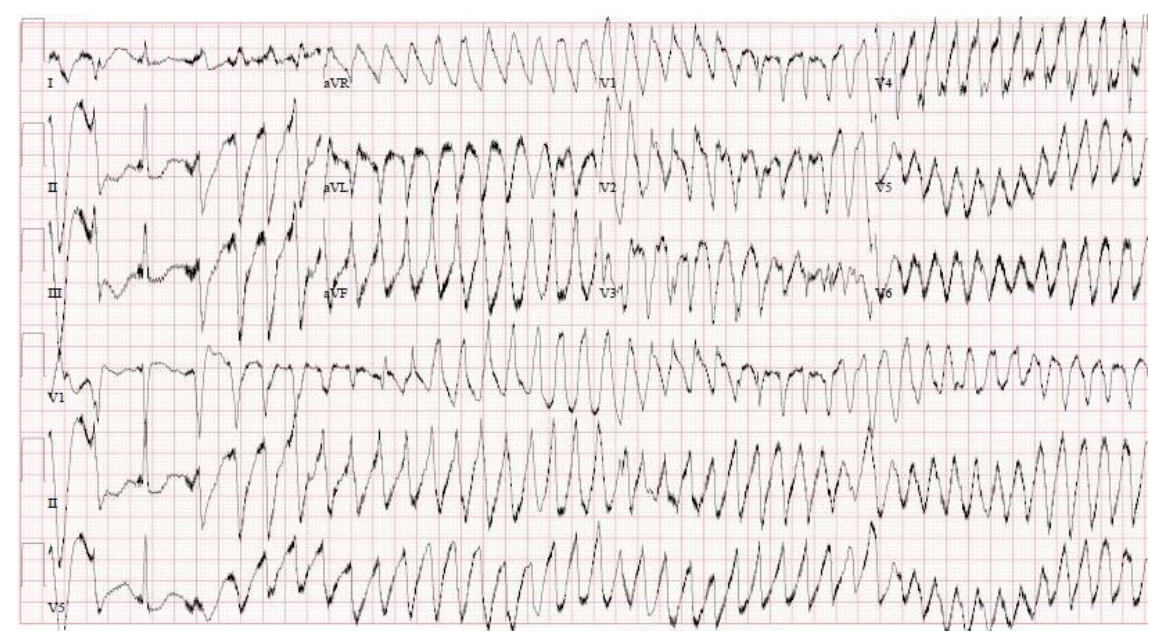

Figure 1: ECG showing distinct TdP. 
terenol infusions were started, after which the patient stabilized. Lidocaine was initiated as a $100 \mathrm{mg}$ IV bolus followed by a $2 \mathrm{mg} / \mathrm{min}$ continuous infusion. Isoproterenol was initiated as a $3 \mathrm{mcg} / \mathrm{min}$ continuous infusion to be titrated between $0.5 \mathrm{mcg} / \mathrm{min}$ and $10 \mathrm{mcg} / \mathrm{min}$ to keep the heart rate above $110 \mathrm{bpm}$. During this time a temporary transvenous pacemaker was considered for overdrive pacing but was not needed due to the quick effectiveness of the isoproterenol. Due to the emergent nature of the event, therapeutic anticoagulation was not initiated prior to defibrillation.

The patient did not experience any additional VT episodes during her hospitalization but required significant electrolyte replacement throughout for a goal potassium above $4 \mathrm{mmol} / \mathrm{L}$ and a goal magnesium above $2 \mathrm{mg} / \mathrm{dL}$, likely due to her chronic alcoholism and poor nutrition at baseline. Repeat ECG the following morning was significant for sinus tachycardia secondary to isoproterenol, but QT prolongation and ventricular arrhythmias had resolved. Lidocaine was infused at a constant rate of $2 \mathrm{mg} / \mathrm{min}$. A random level 26 hours after the start of the lidocaine infusion returned at $3.8 \mathrm{mcg} /$ $\mathrm{mL}$. Liver function tests were minimally elevated during admission (aspartate aminotransferase (AST) 84 unit/L, alanine aminotransferase (ALT) 33 unit/L, total bilirubin $0.9 \mathrm{mg} / \mathrm{dL}$, alkaline phosphatase (alk phos) 82 uni$\mathrm{t} / \mathrm{L}$ ) and remained stable. The patient reported that she had elevated liver function tests (AST 223 unit/L, ALT 84 unit/L, total bilirubin $0.8 \mathrm{mg} / \mathrm{dL}$, alk phos 106 unit/L) 3 months prior to this admission. Left ventricular ejection fraction was slightly decreased at $46 \%$ immediately following her arrest but recovered to $59 \%$ three days later. Left ventricle and left atrium were normal size with no other abnormalities identified on echocardiography and no evidence of intra-cardiac thrombus. No prior echocardiograms were available for comparison. Isoproterenol and lidocaine were successfully discontinued after 2 days of treatment.

The patient was discharged after 4 days of hospitalization with fetal heart sounds intact. She received counseling regarding her alcoholism and its contribution to significant risk of future arrhythmias secondary to electrolyte abnormalities. Prior to admission she was taking two QT prolonging medications, fluoxetine $40 \mathrm{mg}$ daily and trazodone $50 \mathrm{mg}$ daily. These were discontinued on admission with instructions for her to not restart them upon discharge home. Well after the time of discharge at 35 weeks into her pregnancy, her echocardiogram demonstrated a left ventricular ejection fraction recovered to $62 \%$ with mild to moderate mitral valve regurgitation and no other abnormalities. Her fetus was diagnosed with tetralogy of Fallot (TOF) around 25 weeks gestation. The 35 week repeat fetal echocardiogram showed a large ventricular septal defect but normal pulmonary valve without significant subpulmonary obstruction, and it was thought she would likely have a pink TOF. Any impact of the medications on the fetus would be conjecture but maternal alcohol use while pregnant is a known risk factor for TOF [6] and exposure to isoproterenol and lidocaine are not.

\section{Conclusions}

This patient received standard guideline recommended magnesium and defibrillation for treatment of VT in pregnancy, which were unsuccessful in permanently terminating her arrhythmia. IV amiodarone has been reported to cause congenital abnormalities and should be avoided if possible, therefore limiting our therapeutic options $[1,7]$. Lidocaine carries a pregnancy category $B$ rating based on rat reproduction studies with no controlled trials in pregnant humans [8]. Risks with lidocaine include maternal hypotension, fetal bradycardia, and alterations in the central nervous system, vascular tone, and cardiac function [9]. Isoproterenol carries a pregnancy category $C$ because neither animal or human studies have been conducted, and it is suspected to interfere with uterine contractions at term due to beta- 2 agonism $[9,10]$. Despite the potential risks, we found it necessary to chemically terminate this life-threatening, refractory arrhythmia. Since this patient was not close to term, isoproterenol was considered a reasonably safe option. Lidocaine has also been well tolerated in pregnant women and carries less fetal risk than amiodarone, making these two options the most ideal.

Isoproterenol is an IV infusion dosed at $2-10 \mathrm{mcg} /$ min, titrated to heart rate and rhythm response [11]. The lowest effective dose should be used to avoid adverse effects. Lidocaine is given as a $1 \mathrm{mg} / \mathrm{kg}$ bolus followed by an IV infusion dosed at $0.5-4 \mathrm{mg} / \mathrm{min}$, titrated to rhythm response. Plasma lidocaine levels may be obtained, primarily for adverse effect monitoring and prevention. Adverse effects become more likely with levels above $6 \mathrm{mcg} / \mathrm{mL}[8,12]$. Patients with severe hepatic dysfunction are at increased risk of lidocaine toxicity, and those with cardiovascular dysfunction may have decreased ability to compensate for functional changes associated with atrioventricular conduction prolongation [8]. Lidocaine has an estimated volume of distribution of $1.5 \mathrm{~L} / \mathrm{kg}$ and is $60-80 \%$ bound to protein [8]. Pregnancy significantly alters drug pharmacokinetics via a $40 \%$ increase in plasma volume, a decrease in plasma proteins, and augmented hepatic metabolism [13]. It is unknown if alternative dosing strategies should be considered when using lidocaine and isoproterenol in pregnant women. The doses used in our case were not adjusted for pharmacokinetic changes related to pregnancy or potential hepatic dysfunction. We used standard doses, monitoring her clinical response for efficacy and serum lidocaine level for toxicity. A serum lidocaine level obtained 26 hours after the start of the infusion returned at $3.8 \mathrm{mcg} / \mathrm{mL}$, which is within the therapeutic range of $1.5-5 \mathrm{mcg} / \mathrm{mL}$ and below toxic concentrations of $6 \mathrm{mcg} / \mathrm{mL}$. This unique case of TdP and recurrent VT arrests in a pregnant 32-year-old female demonstrates 
efficacy of isoproterenol pacing combined with the antiarrhythmic lidocaine for terminating refractory $\mathrm{TdP}$ and life-threatening ventricular arrhythmia.

\section{Authors Contribution}

All authors contributed equally to this project.

\section{Institution Where Work Completed}

Intermountain Medical Center, 5121 Cottonwood St, Murray, UT 84107, USA.

\section{Conflicts of Interest/Financial Disclosures}

None declared.

\section{Funding Source}

This research did not receive any specific grant from funding agencies in the public, commercial, or not-forprofit sectors.

\section{References}

1. Zipes DP, Camm AJ, Borggrefe M, Buxton AE, Chaitman $B$, et al. (2006) ACC/AHA ESC 2006 guidelines for management of patients with ventricular arrhythmias and the prevention of sudden cardiac death-executive summary. Circulation 114: 1088-1132.

2. Thomas SHL, Behr ER (2016) Pharmacological treatment of acquired QT prolongation and torsades de pointes. $\mathrm{Br} \mathrm{J}$ Clin Pharmacol 81: 420-427.

3. Takaya T, Okamoto M, Yodoi K, Hata K, Kijima Y, et al. (2009) Torsades de pointes with QT prolongation related to donepezil use. J Cardiol 54: 507-511.

4. Katz KD, Cannon RD, Cook MD, Amaducci A, Day R, et al. (2017) Loperamide-induced torsades de pointes: A case series. J Emerg Med 53: 339-344.

5. Mittadodla PS, Salen PN, Traub DM (2012) Isoproterenol as an adjunct for treatment of idiopathic ventricular fibrillation storm in a pregnant woman. Am J Emerg Med 30: 251.e3251.e5.

6. Yang J, Qiu H, Qu P, Zhang R, Zeng L, et al. (2015) Prenatal alcohol exposure and congenital heart defects: A meta-analysis. PLoS One 10: 1-15.

7. (2004) Amiodarone. Philadelphia, Wyeth Pharmaceuticals, USA.

8. (2010) Lidocaine. Schaumburg, APP Pharmaceuticals, USA.

9. (2013) Isoproterenol. Hospira, USA.

10. Mahon WA, Reid DWJ, Day RA (1967) The in vivo effects of beta adrenergic stimulation and blockade on the human uterus at term. J Pharmacol Exp Ther 156: 178-185.

11. Neumar RW (2010) 2010 American heart association guidelines for cardiopulmonary resuscitation and emergency cardiovascular care science. Circulation 122: 750.

12. Al-Khatib S, Stevenson WG, Ackerman MJ, Bryant WJ, Callans DJ, et al. (2017) 2017 AHA/ACC/HRS guideline for management of patients with ventricular arrhythmias and the prevention of sudden cardiac death. Circulation 000: e000-e000.

13. Feghali M, Venkataramanan R, Caritis S (2015) Pharmacokinetics of drugs in pregnancy. Semin Perinatol 39: 512519. 